\title{
Biomarker-Based Treatment Selection in Non-Small Cell Lung Cancer
}

Presented by Wallace Akerley, MD

Abstract
Patients with non-small cell lung cancer must be tested for biomarkers. Currently, treatments directed against EGFR, ALK, and ROS1 muta-
tions are standard of care. A number of emerging new targets and treatments are on the horizon. J Natl Compr Canc Netw 2017;15(5.5):689-691

\begin{abstract}
"This is a very exciting time [in treating lung cancer]. We have always sought to use the right tool for the right job, with the focus placed on making better tools. We just kept varying the chemotherapy combinations leading to a plateau in efficacy. Now we have shifted emphasis toward identification of the 'job' and using the right 'tool' by way of biomarkers," said Wallace Akerley, MD, Director, Thoracic Program, Huntsman Cancer Institute at the University of Utah, and member of the NCCN Non-Small Cell Lung Cancer (NSCLC) Panel. Dr. Akerley reviewed biomarker-based treatment selection at the NCCN 22nd Annual Conference.

NSCLC is a broad term for a collection of cancers of lung origin with different molecular drivers and different ways of circumventing the immune system. Molecular markers such as epidermal growth factor receptor (EGFR), anaplastic lymphoma kinase $(A L K)$, and ROS1 are now required in the 2017 NCCN Clinical Practice Guidelines in Oncology (NCCN Guidelines) for testing in stage IV NSCLC, in addition to other actionable mutations. Currently, immune testing for programmed death ligand-1 (PDL1) is required at diagnosis of stage IV NSCLC to identify a more immune-responsive population for consideration of pembrolizumab treatment.
\end{abstract}

\footnotetext{
Presented by Wallace Akerley, MD, Huntsman Cancer Institute at the University of Utah, Salt Lake City, Utah.

Dr. Akerley has disclosed that he has received grant/research support from ARIAD Pharmaceuticals, Inc, Bristol-Myers Squibb Company, Genentech, Inc., and Novartis Pharmaceuticals Corporation.

Correspondence: Wallace Akerley, MD, Huntsman Cancer Institute at the University of Utah, 2000 Circle of Hope, Salt Lake City, UT 84112. E-mail: wallace.akerley@hci.utah.edu
}

"We don't do immune panels yet, but I expect that in the near future we will be testing for several biomarkers as we better understand the role of the immune system in cancer," Dr. Akerley predicted.

For the purposes of first-line therapy, biomarkers divide NSCLC into 3 groups: (1) those with moleculardefined actionable targets, (2) PD-L1-defined immunosensitive populations, and (3) those fitting neither group who are further categorized by histology as squamous (p63) versus nonsquamous (TTF1). For squamous cell cancers, a platinum doublet excluding pemetrexed is preferred; whereas for nonsquamous cancers, a platinum doublet or triplet with pemetrexed and/or bevacizumab are good choices.

Approximately two-thirds of NSCLC tumors (64\%) will have a driver mutation, among which approximately $25 \%$ are actionable with available therapies. EGFR accounts for approximately $17 \%$, ALK for $8 \%$, and ROS 1 for $1 \%$. Approximately $17 \%$ of patients will have a KRAS mutation, but there is no effective drug yet targeted to that mutation. ${ }^{1}$ Molecular targets with emergent treatment pathways include HER2, MET amplification, $B R A F^{\mathrm{V} 600 \mathrm{E}}$, and RET.

Dr. Akerley stated, "Multiplex panels to identify all actionable mutations are critical to the care of patients with NSCLC because these mutations afford very effective treatment, can only be found if evaluated, and will show increasing prevalence in clinic as effective treatments prolong their survival over those without targetable mutations. For example, a review of one clinic day last week at Huntsman Cancer Institute at the University of Utah showed that 8 of 12 patients 
under treatment for metastatic NSCLC had actionable mutations. These included 5 of $7 \mathrm{NCCN}$ defined actionable mutations: EGFR, ALK, MET, RET, and BRAF."

EGFR is the most frequent of the actionable mutations in NSCLC. It occurs more frequently in Asians (>30\% vs $15 \%$ in whites) and never-smokers (38\% vs $5 \%$ in current smokers). Three generations of tyrosine kinase inhibitors (TKIs) are approved to treat EGFRbiomarker-defined NSCLC. Among those with mutations, 90\% will have a deletion in exon 19 or L858R in exon 21 of EGFR, Dr. Akerley said.

In the early days when EGFR was not yet recognized as the biomarker for response to EGFR inhibitors, a study enriched for those likely to respond to EGFR therapy (Asians and never-smokers) showed that those with EGFR-positive NSCLC had significantly superior progression-free survival (PFS) on treatment with gefitinib (an EGFR inhibitor) versus carboplatin/paclitaxel $(P<.001)$, whereas those who were EGFR-negative had significantly improved PFS on carboplatin/paclitaxel versus gefitinib $(P<.001){ }^{2}$

"This early study showed that PFS was significantly improved if you give the right drug to the right patient and established biomarker-directed therapy of NSCLC," Dr. Akerley stated. "EGFR mutation is predictive for response and prognostic for survival. In my opinion, EGFR-mutated and EGFR wild-type NSCLC are 2 discrete cancers, as different as breast cancer and colon cancer," he said.

"All EGFR mutations are not created equal. First-generation TKIs block EGFR sensitivity mutations (exons 19 and 21) of the cancer, as well as the wild-type EGFR of the skin and gut, which accounts for the most common side effects. Osimertinib, a third-generation TKI, affects the same EGFR sensitivity mutations plus the T790 mutation, the acquired mutation that is the most common cause of eventual resistance to first- and second-generation TKIs," he said. Additionally, it has less effect on wild-type EGFR of the gut and skin, so adverse effects are minimized.

\section{Osimertinib}

Osimertinib has been changed from a category 2A recommendation to a category 1 recommendation for patients with metastatic NSCLC in the 2017 NCCN Guidelines.

A study of second-line treatment of T790Mresistant stage IV NSCLC found that osimertinib achieved a response rate of $62 \%$, duration of response of 15.2 months, and intracranial response of $64 \%{ }^{3}$ This drug is an irreversible inhibitor and crosses the blood-brain barrier, accounting for its intracranial effectiveness. ${ }^{4}$

A recent randomized study in T790M-resistant NSCLC found that second-line treatment with osimertinib improved median PFS to 10.1 months versus 4.4 months with platinum-based chemotherapy $(P<.001)$. In a subgroup of patients with brain metastases, osimertinib improved PFS to 8.5 months from 4.2 months with chemotherapy. Adverse events of grade 3 or 4 occurred in twice as many patients on chemotherapy versus osimertinib (23\% vs $47 \%$, respectively). ${ }^{5}$ Approximately $49 \%$ of resistance to TKI therapy is accounted for by T790M.

\section{ALK Mutations}

ALK is another common mutation in NSCLC occurring in $4 \%$ to $5 \%$ of NSCLCs. Three agents are FDA-approved for the treatment of ALK-positive disease: crizotinib, ceritinib, and alectinib. Other new agents look promising, Dr. Akerley said.

In a phase III study of ALK-positive NSCLC, crizotinib doubled PFS compared with chemotherapy (pemetrexed or docetaxel). Median PFS was 7.7 versus 3 months, respectively $(P<.001)$, whereas overall survival was similar between treatment arms. ${ }^{6}$ It has been the only ALK-targeted therapy for the first-line treatment of NSCLC.

Ceritinib is FDA-approved as second-line treatment for ALK-positive NSCLC, and has been added as a category 1 recommendation in the $2017 \mathrm{NCCN}$ Guidelines as first-line treatment of ALK-translocated NSCLC. In the recent ASCEND-4 phase III study, median PFS was superior with first-line ceritinib versus pemetrexed/cisplatin chemotherapy (16.6 vs 8.1 months, respectively; $P<.00001) .{ }^{7}$ Subgroup analysis showed median PFS of 26.3 versus 8.3 months, respectively, in patients with no brain metastases, and a median of 10.7 versus 6.7 months in those with brain metastases. Overall survival is not yet mature in this trial, but thus far, the numbers suggest that survival may be improved with ceriitinib. 
Alectinib is the third drug approved for ALKpositive NSCLC. In a phase II trial of patients with crizotinib-resistant NSCLC, the response rate was $55 \%$ (originally reported as $48 \%$ ). Central nervous system penetration was seen in $75 \%$ of 16 patients with brain metastases. ${ }^{8}$

The first report from the J-ALEX trial, that is comparing alectinib versus crizotinib in ALK-positive NSCLC in an Asian population who had 0 or 1 previous therapies, showed superior PFS for alectinib versus crizotinib (not yet reached vs median 10.2 months, respectively; P<.0001). " "So this study appears to show that alectinib is superior to crizotinib in an Asian population with NSCLC," Dr. Akerley said. Results of a similar study comparing crizotinib versus alectinib in a general population of ALK-rearranged NSCLC (ALEX) will be reported soon to help refine the best sequence of ALK biomarker-defined therapy.

"There are a number of approved and effective ALK therapies at this time, and the choice of firstor second-line therapy is largely directed by toxicity. While effective, these agents have differing spectrums of sensitivity, and treatment will likely cause different resistance mutations to emerge. In the future, it is probable that serial sampling of ALK-rearranged tumors will identify biomarkers to determine the most effective ALK therapy initially and at time of resistance," he stated.

\section{Other Less Frequent Mutations and Emerging Therapies}

ROS1 rearrangement-positive cancers have a new page in the 2017 NCCN Guidelines for NSCLC. Crizotinib is currently suggested as the treatment option for this group of tumors (category $2 \mathrm{~A}$ recommendation).

The NCCN Guidelines list several actionable genetic mutations and emerging strategies to treat patients with those gene alterations. These include RET rearrangements (cabozantinib, vandetanib), $B R A F^{\mathrm{V} 600 \mathrm{E}}$ mutations (vemurafenib, dabrafenib, and dabrafenib + trametinib), MET amplification or exon 14 skipping mutation (crizotinib), and HER2 mutations (trastuzumab and afatinib; both category 2B).

"Although all of these drugs are FDA-approved for various cancers, most do not yet have an approved indication for NSCLC. Nevertheless, adequate published data support their efficacy for biomarkerdefined NSCLC, and it would be unreasonable for an oncologist to not use these agents when the mutation has been identified," Dr. Akerley said.

"Biomarkers identify commonalities of cancer mechanisms and, therefore, maximize the probability of treating patients most effectively. Clinical trials have advanced this strategy to the standard of care in NSCLC. Phase I trials afford the opportunity for access to new molecularly targeted therapies, so their integration into everyday cancer care can have the potential to radically impact patient outcomes. Broad molecular testing is the keystone of biomarkerdirected therapy of NSCLC," Dr. Akerley said.

\section{References}

1. Kris MG, Johnson BE, Berry LD, et al. Using multiplexed assays of oncogenic drivers in lung cancer to select targeted drugs. JAMA 2014;311:1998-2006.

2. Mok $\mathrm{T}, \mathrm{WuY}$, Yang $\mathrm{CH}$, et al. Gefinitib or carboplatin-paclitaxel in pulmonary adenocarcinoma. N Engl J Med 2009;361:947-957.

3. Jänne PA, Yang JC, Kim DW, et al. AZD9291 in EGFR inhibitor-resistant non-small cell lung cancer. N Engl J Med 2015;372:1689-1699.

4. Yang JC, Ahn MJ, Kim DW, et al. Osimertinib in pretreated T790Mpositive advanced non-small-cell lung cancer: AURA study phase Il extension component [published online ahead of print February 21, 2017] J Clin Oncol, doi: 10.1200/JCO.2016.70.3223.

5. Mok T, Wu YI, Ahn MJ, et al. Osimertinib or platinum pemetrexed in EGFR T790M-positive lung cancer. N Engl J Med 2017;376:629-640.
6. Shaw AT, Kim DW, Nakagawa K, et al. Crizotinib versus chemotherapy in advanced ALK-positive lung cancer. N Engl J Med 2013;368:23852394.

7. Soria JC, Tan CS, Chiari R, et al. First-line ceritinib versus platinumbased chemotherapy in advanced ALK-rearranged non-small-cell lung cancer (ASCEND-4): a randomized, open-label, phase 3 study. Lancet 2017;389:917-929.

8. Shaw AT, Gandhi L, Gadgeel S, et al. Alectinib in ALK-positive, crizotinibresistant, non-small-cell lung cancer: a single-group, multicenter, phase 2 trial. Lancet Oncol 2016;17:234-242.

9. Nokihara H, Hida T, Kondo M, et al. Alectinib versus crizotinib in ALKinhibitor naïve AL-positive non-small cell lung cancer: primary results from the J-ALEX study [abstract]. J Clin Oncol 2016:34(Suppl):Abstract 9008 . 